\title{
The Bondability and Reliability of a Ternary Ag Alloy Wire on an Al Bonding Pad under $\mathbf{N}_{2}$-Free Conditions
}

\author{
Gun Rae Kim ${ }^{1,2}$, Sang-Su Ha ${ }^{1}$, Cheol-Hyeon Park ${ }^{1}$, Sangwoo Pae ${ }^{1}$ and Byoungdeog Choi ${ }^{2 * *}$ \\ ${ }^{1}$ Technology Quality and Reliability, Quality and Reliability Team, Foundry Business, Samsung Electronics Co., Ltd., \\ San \#24, Nongseo-Dong, Giheung-Gu, Yongin, Gyeonggi-Do 17113, Korea \\ ${ }^{2}$ School of Information and Communication Engineering, Sungkyunkwan University, Suwon 16419, Korea
}

\begin{abstract}
Generally, high-purity $99.99 \%$ Au wires are mainly used for electrical connection to transmit and receive data between an Al bonding pad and a printed circuit board (PCB) substrate in semiconductor devices. However, there have been several studies to reduce the cost of expensive $\mathrm{Au}$ wires due to the sharp increase in the price of $\mathrm{Au}$. Thus, $\mathrm{Ag}$ and $\mathrm{Cu}$ alloy wires have been mainly considered as alternative materials.

This paper focused on a ternary Ag alloy wire (Au: 1.5\%, Pd: $2.5 \%$ ) and explained free air ball (FAB) shape effects with various electronic flame-off (EFO) conditions in more detail and experimentally showed the susceptibility to bonding failure of the Ag alloy wire after exposure to the atmosphere at room temperature. In particular, a feasible $\mathrm{Ag}$ wire bonding process and grain structure effects under $\mathrm{N}_{2}$-free conditions were studied in depth for the first time. With this $\mathrm{N}_{2}$-free condition, weak surface tension of a melting wire led to move FAB quickly to the interface between FAB and heat affected zone (HAZ). In addition, a slender grain structure in the center of FAB area still remained because of the short time current stressing. It made good conductivity and no significant resistance change of a Ag alloy wire. For a $\mathrm{Ag}$ alloy wire exposed to the atmosphere for 19 days, the defective percentage during bonding process suddenly started to rise, and reached more than $0.7 \%$ in 24 days. According to our results, less than 18 days are highly recommended as a control standard of a Ag alloy wire when it is exposed to the atmosphere. The bonding between a Ag alloy wire and an Al bonding pad was formed without any crack in the lower part of an Al bonding pad. Higher intermetallic compound coverage and minimum Al splash were chosen as key bonding parameters, and $\mathrm{KOH}$ cratering tests were performed to secure good bonding quality. Finally, robust Ag wire reliability has been successfully achieved and demonstrated. [doi:10.2320/matertrans.MG201803]
\end{abstract}

(Received April 4, 2018; Accepted June 8, 2018; Published July 23, 2018)

Keywords: $\mathrm{Ag}$ wire, ternary, $\mathrm{N}_{2}$ free, corrosion, oxidation, bondability, splash, reliability

\section{Introduction}

High-purity $99.99 \%$ Au wires have been widely used for electrical connections between $\mathrm{Al}$ bonding pads for the power supply and external substrate terminals PCB (printed circuit board), lead frame, and others in semiconductor devices. Because of the increased price of $\mathrm{Au}$ wires and the trend of cost reduction in semiconductor devices, $\mathrm{Cu}, \mathrm{Ag}$, and $\mathrm{Pd}$ wires have been considered as alternative materials. However, they have limited application to some products and their widespread use is difficult due to concerns over reliability and the risks associated with several problems, such as wire oxidation, and the challenge of determining the optimum bonding parameters between the wires and Al bonding pads.

Methods for saving on the cost of $\mathrm{Au}$ wires have been developed, including reducing the wire diameter and creating new materials with the addition of alloy elements. Recently, $\mathrm{Au}$ wire diameters have been reduced to $13 \mu \mathrm{m}$. However, semiconductor products were confronted through a decrease in speed, wire sweeping in the package mold process, and the lack of an operating margin in module implementation when using Au wires with a reduced diameter. Therefore, a large number of studies on Ag to develop inexpensive alloy wires have emerged and actively studied as alternatives. ${ }^{1)}$

Table 1 shows some general material properties. ${ }^{2)}$ As shown in Table 1, Cu wire not only has excellent thermal and electrical characteristics, but also a higher elongation strength via the mechanical force. These superior properties of $\mathrm{Cu}$ make the distance between the ball and wedge bonds longer, which is favored from a sweeping wire perspective. ${ }^{2-6)}$

*Corresponding author, E-mail: bdchoi@skku.edu
As mentioned above, while $\mathrm{Cu}$ bonding wire has advantages over Au bonding wire in some aspects, it has not been widely used. The reason is that $\mathrm{Cu}$ wire is much harder than $\mathrm{Au}$ bonding wire, and it can cause damage induced by higher ultrasonic power under the Al metallization pad. Additionally, $\mathrm{Cu}$ wire can be easily oxidized on a surface, resulting in poor bondability to wedge bonding due to the surface oxidation. In this research, we investigate and present $\mathrm{Ag}$ wire as a promising new bonding wire material. Because the Young's modulus and Vickers hardness is low, Ag wire has less impact on the $\mathrm{Al}$ bonding pad than $\mathrm{Cu}$ wire. $\mathrm{Pd}$ - and Au-doped $\mathrm{Ag}$ alloy wires both increase the mechanical strength and improve its oxidation and corrosion resistance. Adding Pd also reduces the electromigration of the pure $\mathrm{Ag}$ wire and inhibits the overgrowth of intermetallic compounds at the interface between the Ag wire and Al bonding pad. ${ }^{7)}$ For incentive, $\mathrm{Ag}$ has superior excellent thermal conductivity and electrical properties, and it leads to a meaningful cost reduction compared with $\mathrm{Au}$ wire because it is $1 / 20$ cost of Au. ${ }^{8)}$

For the first time, this study evaluates the bonding properties of ternary Ag alloy wires and especially presents the effect of $\mathrm{N}_{2}$-free conditions on the Ag alloy wires during the EFO process. Ultimately, it is expected that these verification methods will contribute to the stabilization of the bonding quality and create cost-saving effects during mass production.

\section{Experimental Procedure}

\subsection{Wire bonding process and analysis}

A ternary $\mathrm{Ag}$ alloy (Au: $1.5 \%, \mathrm{Pd}: 2.5 \%$ ) wire with an $18 \mu \mathrm{m}$ diameter was used in our investigation. To develop a 
Table 1 Physical properties of pure metal. ${ }^{2)}$

\begin{tabular}{c|c|c|c}
\hline Wire material & Ag & Au & Cu \\
\hline Atomic weight $(\mathrm{g} / \mathrm{mol})$ & 108 & 197 & 64 \\
\hline Density $\left(\mathrm{g} / \mathrm{cm}^{3}\right)$ & 10.5 & 19 & 1085 \\
\hline Melting point $\left({ }^{\circ} \mathrm{C}\right)$ & 961 & 1064 & $1.72 \times 10^{-8}$ \\
\hline Electrical resistivity $(\mathrm{ohm} \cdot \mathrm{m})$ & $1.63 \times 10^{-8}$ & $2.21 \times 10^{-8}$ & 400 \\
\hline Thermal conductivity $(\mathrm{W} / \mathrm{mk})$ & 430 & 78 & 130 \\
\hline Young's modules $(\mathrm{GPa})$ & 83 & 216 & 369 \\
\hline Vickers hardness $(\mathrm{Mpa})$ & 251 & & 780 \\
\hline
\end{tabular}

good shape of free air ball (FAB) for a stable wire bonding process, it is essential to optimize the electronic flame-off (EFO) condition. An unoptimized EFO condition could make off-centered FAB, long-tailed FAB, or small-sized FAB, which could cause bonding failure. In this work, bonding processes were achieved using ICONN (KNS Co. Ltd.), and Al metallization was used for the bonding pad.

While processing to form ball bonding of the Ag alloy wire, surface oxidation arises in FAB after EFO. To avoid oxidation, nitrogen gas is conventionally used. However, in this work, nitrogen gas was not used $\left(\mathrm{N}_{2}\right.$ free) for the first time for different reasons including cost-saving effect. Abnormal FAB shape leads to bonding failure, so it is monitored and detected by a sensor equipped with ICONN.

The bonding failure rate shown in Fig. 4(a) was calculated as the number of failures divided by the number of bonding processes. To obtain the bonding failure rate during wire bonding process as shown in Fig. 4(a), it was performed more than one thousand times per day. The types of bonding failure are categorized in two ways. One is open failure, e.g., non-stacking on lead (NSOL) or non-stacking on pad (NSOP), and the other is EFO error, e.g., small-sized FAB or long-tailed $\mathrm{FAB}$.

High specification auger electron spectroscopy (AES, Model: JAMP-9510F) analysis was performed to measure the atomic percent $(\%)$ of oxygen on the surface of the ternary Ag alloy wire after exposure to the atmosphere at room temperature.

In Fig. 5, an optical scope (Model: Olympus MX50) was used to observe the bottom surface of ball bonding, and intermetallic compound (IMC) coverage was taken by analyzing cross-sectional images with the Imagen software (KNS Co. Ltd).

The cratering test after chemical de-capsulation with $\mathrm{HNO}_{3}$ was performed to identify under-pad damage and the $\mathrm{Al}$ splash by SEM analysis. The Al splash phenomenon occurs due to the wire hardness and unoptimized bonding parameters. ${ }^{6)}$ It results in $\mathrm{Al}$ pad damages, and cracks in the metallic or inter-metal dielectric (IMD) layers can be found. To check these damages, a $45 \% \mathrm{KOH}$ solution was used in order to observe discoloration caused by the deformation. For more information, we measured the whole area of IMC to demonstrate the bonding quality and used more than $70 \%$ bond integrity as the criterion of IMC coverage to the $\mathrm{Al}$ bonding pad.

\subsection{Reliability test conditions and analysis}

To secure the reliability of the ternary $\mathrm{Ag}-\mathrm{Au}-\mathrm{Pd}$ alloy bonding wire, the following test conditions were applied with packaged chips:

(a) High Temperature Storage Test (HTS): 1,000 hours at $150^{\circ} \mathrm{C}$

(b) Pre-condition, Moisture Sensitivity Level (MSL) III: 192 hours at $30^{\circ} \mathrm{C} / 60 \%$ relative humidity.

(c) Temperature Cycle (TC): 1,000 cycles at $-55^{\circ} \mathrm{C} \sim$ $125^{\circ} \mathrm{C}$ (air to air).

(d) Unbiased Highly Accelerated Temperature and Humidity Stress Test (uHAST): 168 hours at $130^{\circ} \mathrm{C} /$ $85 \%$ relative humidity.

(e) Temperature Humidity Bias Test (THB): 1000 hours at $85^{\circ} \mathrm{C} / 85 \%$ relative humidity with $1.4 \mathrm{xVdd}$ bias.

FIB treatment was used for cross-sectional preparation, and then the sample was transferred to SEM for investigation of the interface microstructures.

\section{Results and Discussions}

During the ultrasonic bonding process, consistent size and spherical appearance of FAB are necessary because of large variation. For FAB optimization, it is important to discover the key EFO parameters to form a good FAB shape. In this research, we considered the EFO current as well as the EFO time in the design of experiment (DOE). The relationship between two parameters is shown in Fig. 1, and the FAB height range was from $17 \mu \mathrm{m}$ to $35 \mu \mathrm{m}$ under these conditions. In the case of condition $\mathrm{A}$, a minimum $\mathrm{EFO}$ current was given to wire, and small-sized FAB was formed because of the energy transfer caused by good thermal conduction of the Ag alloy wire to the heat affected zone (HAZ). On the other hand, at the EFO current of condition $\mathrm{D}$, the FAB grows very quickly because of the largest EFO current.

The unoptimized current given to melt the wire results in off-centered or poor FAB shape and ripple, which are two of the major defects during FAB formation. ${ }^{9-13)}$ The spherical shape of FAB is ideally required to ensure good bondability.

From Fig. 2, the relationship between the EFO current and off-centered FAB was observed. As the EFO current increased, the percentage of the off-centered FAB increased and an irregular ball size was also observed. When the EFO current was condition $\mathrm{B}$, no off-centered FAB formation was found. However, an abnormal percentage suddenly increased 


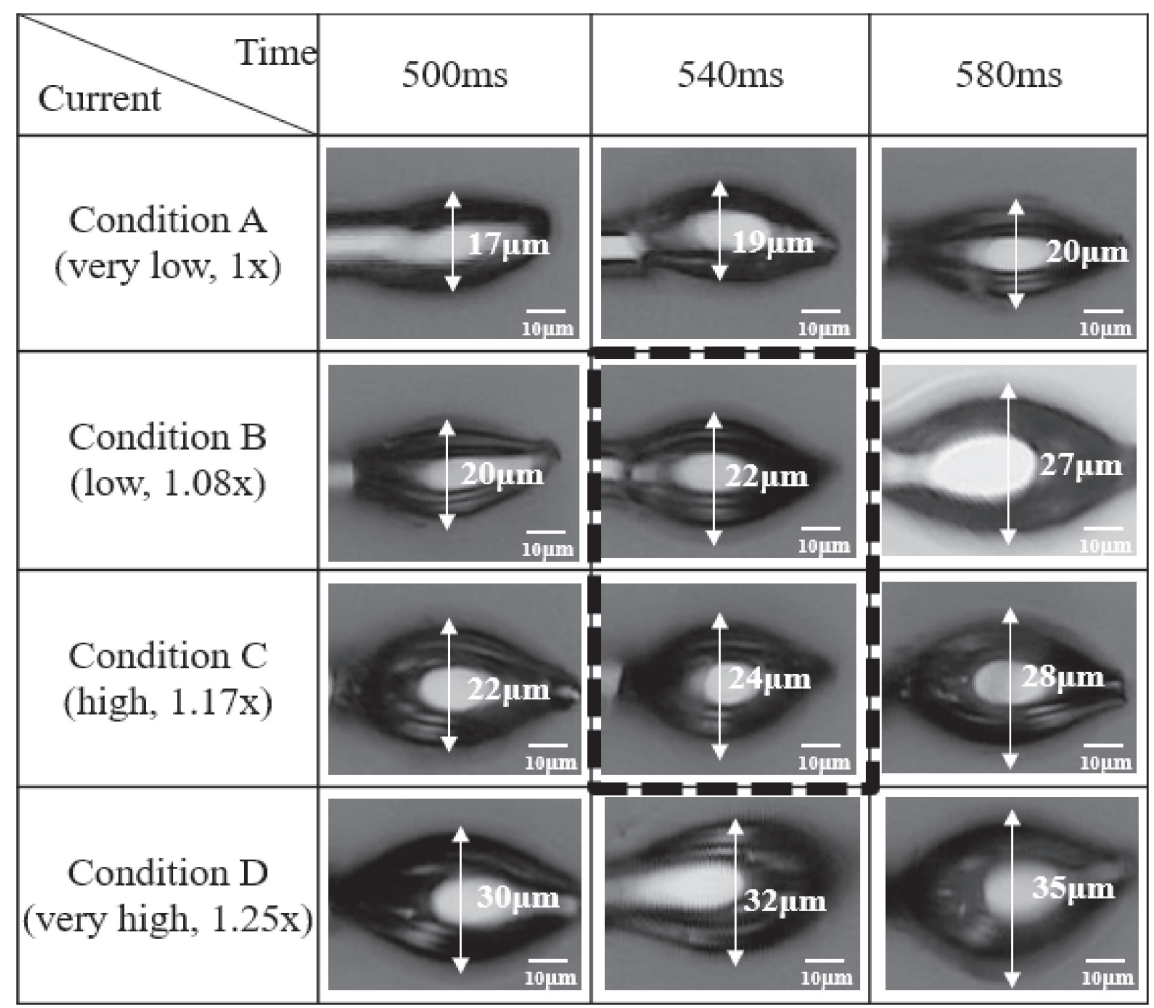

Fig. 1 Free air ball (FAB) formed under different electronic flame-off (EFO) conditions.

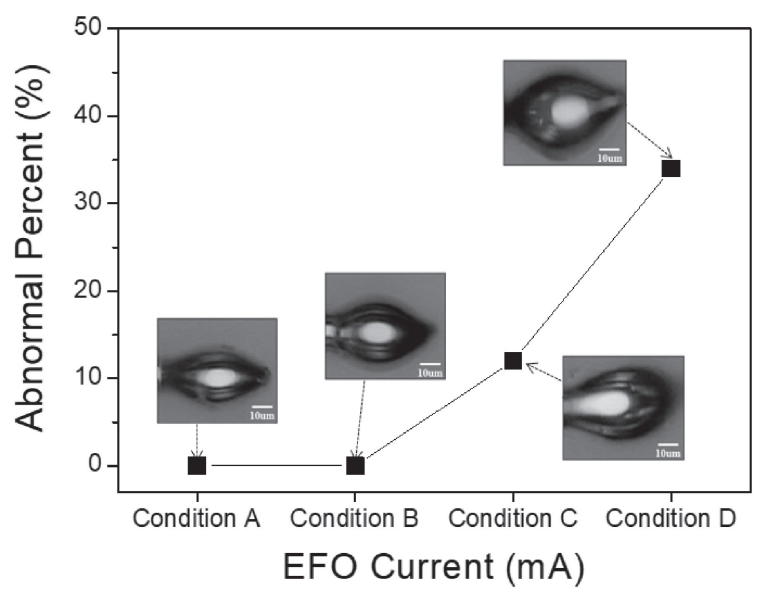

Fig. 2 Off-center percentage of Ag alloy FAB under different EFO currents.

when the EFO current was higher than condition B. In addition, larger ripples also appeared on the surface of the FAB, which could cause a short failure with neighboring wire bonding.

The optimum FAB shape and microstructures of the $\mathrm{Ag}$ alloy wire with the condition of $\mathrm{B}$ after EFO are shown in Fig. 3. In our work, the optimal FAB diameter of the $\mathrm{Ag}$ alloy wire was $22 \mu \mathrm{m}$. When we observe the grain structure in bonding wires, it is obvious that the electrical current raises the wire temperature and leads to a larger grain structure by recrystallization. In Fig. 3(b), we found that the grain size of (2) is larger than (1) in FAB. When we conventionally use $\mathrm{N}_{2}$ gas during the EFO process, wire ripple is formed at the edge of the bonding wire because the strong surface tension. However, without $\mathrm{N}_{2}$ gas, weak
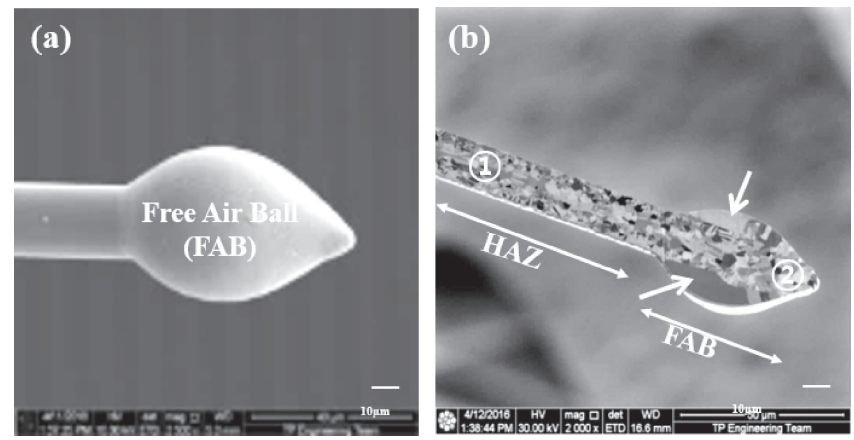

Fig. 3 Optimum FAB shape: (a) SEM and (b) FIB images.

surface tension makes FAB move upward rapidly near the HAZ while the electrical current is applied to the bonding wire. For this reason, a much larger grain structure was found at the top and bottom area in the interface between FAB and the HAZ area as indicated with arrows in Fig. 3(b), because the grain size got larger through recrystallization after the wire melted. Because of the short time current stressing, a slender and small grain structure still remained at (1) and (2) regions. The small grain structure such as a pure Ag alloy wire has good conductivity and small resistance. Note that there is no electrical failure induced by the resistance increase in the long-term reliability test besides manufacturability for this bonding process and ternary Ag alloy wires. For your information, it is quite difficult to directly measure the temperature of a bonding wire, grain structure and measuring its electrical resistivity could be alternate methods for determining the temperature of the bonding wire.

Figure 4(a) shows the results of the failure ratio during the bonding process with two kinds of storage conditions for the $\mathrm{Ag}$ alloy wire, both at room temperature and in a $\mathrm{N}_{2}$ box. 
(a)

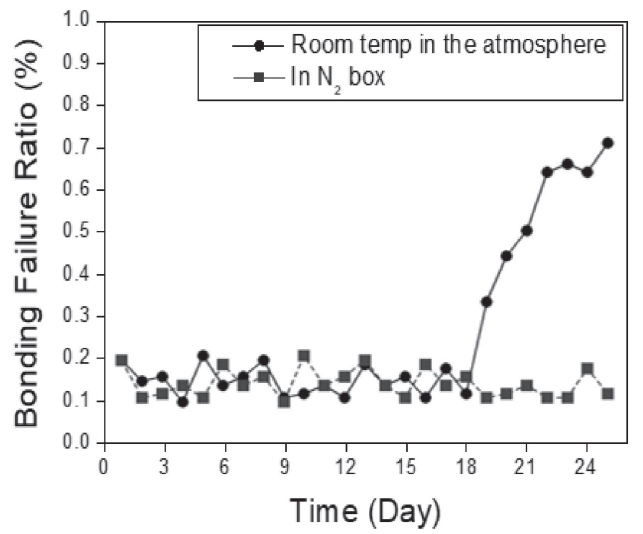

(b)

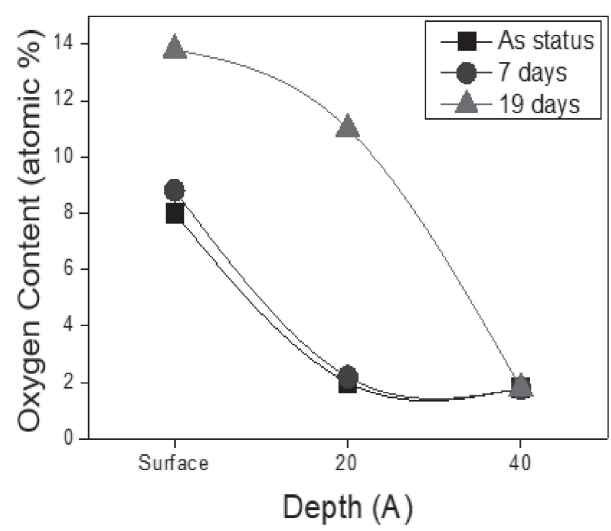

Fig. 4 (a) Bonding failure ratio and (b) AES analysis results after exposure to the atmosphere at room temperature.

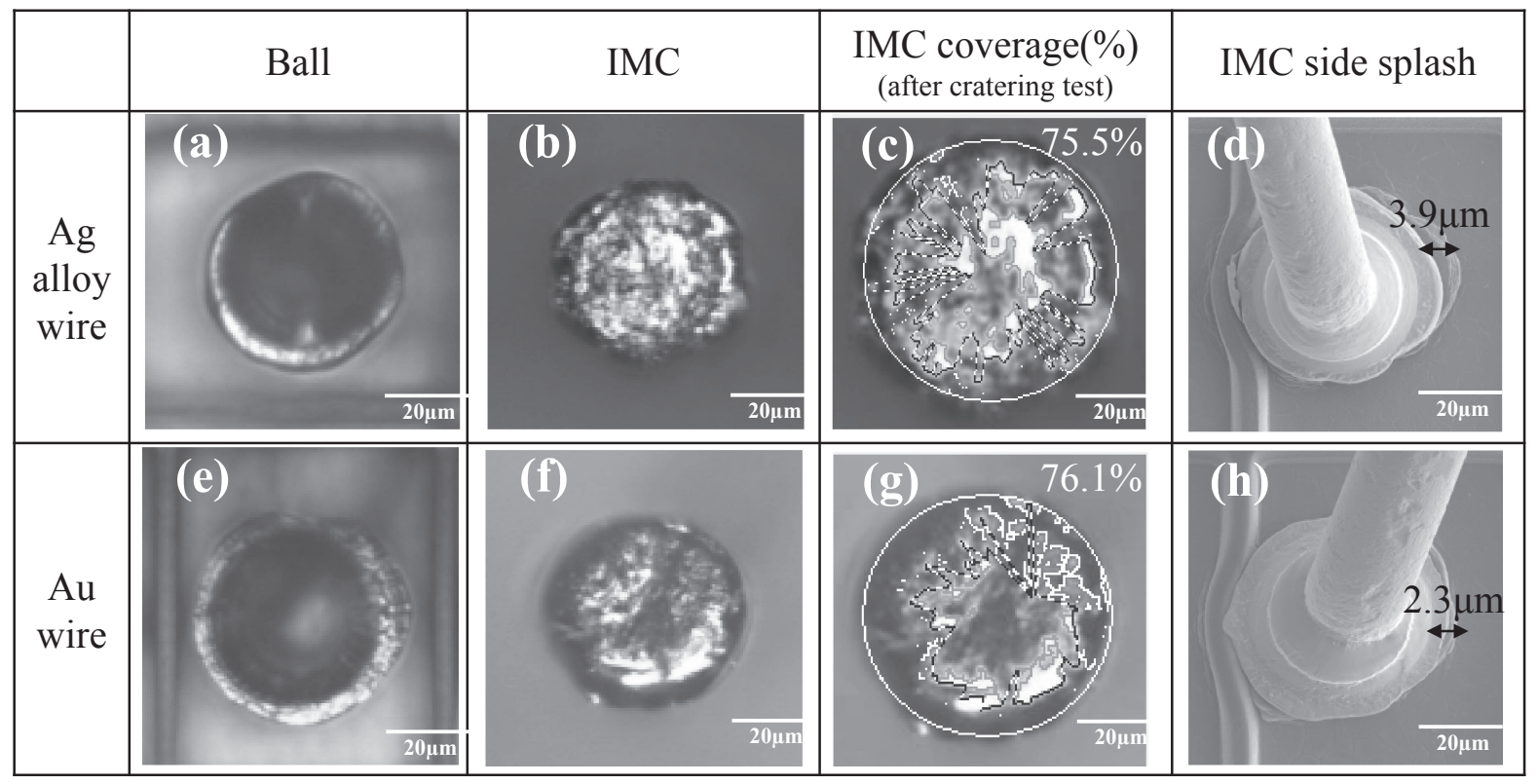

Fig. 5 Optical and SEM images of IMC coverage at the ball bottom surface (b), (c), (f), (g) and side splash (d), (h).

Figure 4(b) shows the oxide atomic percent distribution from the surface after a Ag alloy wire was exposed to the atmosphere at room temperature. Originally, a Ag alloy wire is vacuum-packed.

According to Fig. 4(a), the Ag alloy wires stored in the $\mathrm{N}_{2}$ box show a failure ratio of approximately $0.1 \%$, even after 24 days during the wire bonding process. In contrast, the percent of defective Ag alloy wires stored at room temperature started to rise after 19 days, and it finally reached a defect ratio of more than $0.7 \%$ in 24 days after unpackaged. In order to identify the causes, the oxide content on the surface of the Ag alloy wires was measured, as shown in Fig. 4(b). According to the results, the oxide content on the surface of the Ag alloy wires highly depends on exposure time in the atmosphere. Moreover, an oxide concentration of $20 \%$ from the surface of the Ag alloy wires was detected for long-time exposure in the atmosphere. From these results, less than 18 days could be a control standard when a Ag alloy wire is exposed to the atmosphere. A thin film Ag oxide mentioned above is known as $\mathrm{Ag}_{2} \mathrm{O}$.
Figure 5 presents the SEM image of IMC coverage of the $\mathrm{Al}$ bonding pad. The IMCs above the bonding interface are known as $(\mathrm{Ag}, \mathrm{Au}, \mathrm{Pd})_{2} \mathrm{Al}^{14}{ }^{14}$ More than $75 \%$ IMC coverage was identified in the Ag alloy wire. The superb bonding properties between the $\mathrm{Al}$ bonding pad and $\mathrm{Ag}$ alloy wires were proven. The IMC formation coverage and side splash results after bonding are summarized in Fig. 5(c) and Fig. 5(g). They reveal similar IMC coverage for both wires (Ag alloy wire: $75.5 \%$, Au wire: $76.1 \%$ ).

In fact, the Ag wire highly tends to splash out for high intermetallic compound coverage to a bonding pad because the Ag alloy wire shows $20 \%$ higher hardness than the $\mathrm{Au}$ wire. Higher intermetallic compound coverage as well as minimum Al splash to avoid bridging to the neighboring pad is desired for good bonding quality. The reason for wide usage of $\mathrm{Au}$ wires is that it is easily controlled for wire bonding performance compared to $\mathrm{Ag}$ alloy wires, as stated above.

Figure 6 shows the results of the FIB analysis and comparison of the interface between two different wires and the $\mathrm{Al}$ pad. It is shown that bonding with the $\mathrm{Al}$ pad is 


\section{※ $\mathrm{THK}_{\mathrm{Al} \mathrm{Pad}}$ : Thickness of Al Pad}

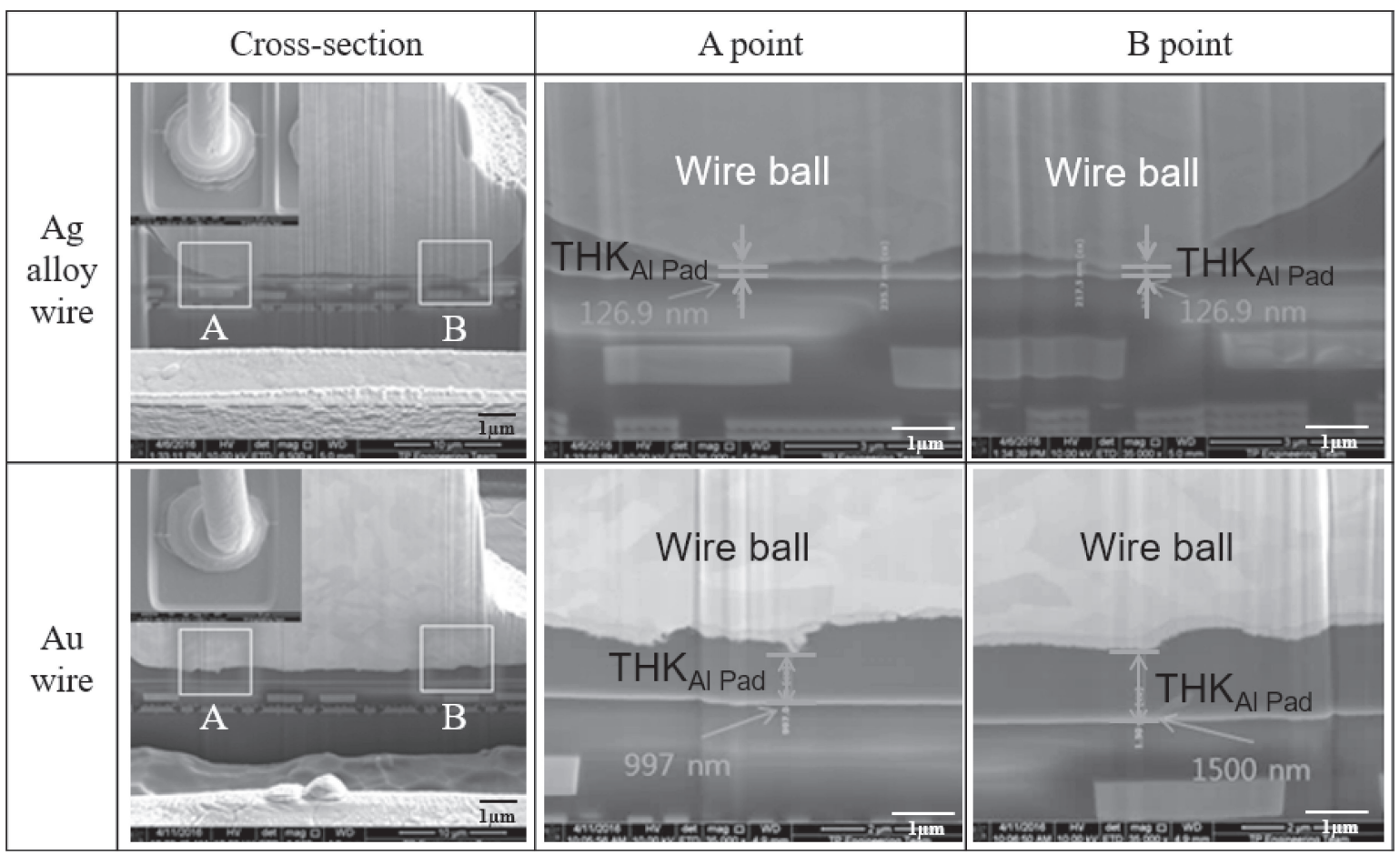

Fig. 6 FIB cross-sectional images of the $\mathrm{Ag}$ and $\mathrm{Au}$ wire joint after bonding on an $\mathrm{Al}$ pad.

Table 2 Reliability test results.

\begin{tabular}{c|c|c}
\hline Reliability test & Test Time & Result \\
\hline HTS $\left(150^{\circ} \mathrm{C}\right)$ & $1,000 \mathrm{hrs}$ & Pass $(0 / 77)$ \\
\hline Precondition $($ Level 3) & $192 \mathrm{hrs}$ & Pass $(0 / 231)$ \\
\hline Temperature cycle $\left(-55 \sim 125^{\circ} \mathrm{C}\right)$ & 1,000 cycles & Pass $(0 / 77)$ \\
\hline uHAST $\left(130^{\circ} \mathrm{C} / 85 \%\right)$ & $168 \mathrm{hrs}$ & Pass $(0 / 77)$ \\
\hline $\mathrm{THB}\left(85^{\circ} \mathrm{C} / 85 \%, 1.4 \mathrm{xVdd}\right)$ & $1000 \mathrm{hrs}$ & Pass $(0 / 77)$ \\
\hline $\mathrm{KOH} \mathrm{Test}$ & To Check pad damage & Pass $(0 / 138)$ \\
\hline
\end{tabular}

well formed without any cracking in the lower part of the $\mathrm{Al}$ metallization pad in both the Ag alloy wire and the Au wire. However, the remaining $\mathrm{Al}$ pad thickness is about $1 \mu \mathrm{m}$ thinner for the Ag alloy wire than for the $\mathrm{Au}$ wire.

Al splash and localized pad thinning is not desired because it can degrade bonding quality. For the purpose of securing the reliability of $\mathrm{Ag}$ wire bonding, various reliability tests were performed with packaged samples. The test results abided by JEDEC standards and details of sample size are listed in Table 2. Finally, all environmental tests, including pre-conditioning level 3 tests, were passed using both an electrical test and scanning acoustic tomography (SAT) to check for any delamination of interfaces within the package.

\section{Conclusion}

In order to examine a cost-effective wire bonding solution, ternary $\mathrm{Ag}$ alloy $(1.5 \% \mathrm{Au}, 2.5 \% \mathrm{Pd}, 96 \% \mathrm{Ag})$ wires were studied to derive the optimum feasible process conditions, especially with $\mathrm{N}_{2}$-free conditions.

When the Ag alloy wires were stored at room temperature after they were released from being vacuum-packed, the amount of oxidation from the wire surface was 14 atomic percent after 19 days of storage. The bonding failure ratio caused by oxidation was stable within 18 days, but rapidly increased and reached more than $0.7 \%$ after 24 days. According to this result, less than 18 days of storage at room temperature is recommended as the maximum period after the Ag alloy wires are unpackaged. This ternary Ag alloy wire showed more than $75 \%$ IMC coverage after bonding, and we found that the bonding strength with the Al metallization pad is sufficient, especially compared with the $\mathrm{Au}$ wire. Based on FIB cross-sectional analysis, because the remaining $\mathrm{Al}$ pad thickness of the Ag alloy wire is about $1 \mu \mathrm{m}$ thinner than that of the Au wires after wire bonding due to the hardness of the Ag alloy wire, it causes difficulties 
during the wire bonding process, and eventually could lead to degradation of the bonding quality.

In this research, the optimal EFO condition was achieved in a $\mathrm{N}_{2}$-free process for the first time and the effect of EFO current and time on FAB was evaluated. This EFO condition makes the grain structure slender similar to a pure ternary Ag alloy wire in FAB.

Therefore, the robust $\mathrm{Ag}$ alloy (1.5\% Au, 2.5\% Pd, 96\% $\mathrm{Ag}$ ) wire reliability has been proved and demonstrated from the reliability stress results from pre-condition, temperature cycle, uHAST, HTS, and THB tests.

\section{REFERENCES}

1) G. Harman: Wire Bonding in Microelectonics; Materials, Processes, Reliability and Yield, (McGraw-Hill, New York, 1997) pp. 1-11.

2) C. Oijia, P. Ariel, R. Dexter, T. Sven and J.T. Howell: Proceedings of the 12th Electronics Packaging and Technology Conference, (2010) pp. 591-596.

3) C. Hang, C. Wang, M. Shi, X. Wu and H. Wang: Proceedings of the 6th International Conference on Electronic Packaging Technology, (2005) pp. 414-418
4) Y.H. Lu, Y.W. Wang, B.K. Appelt, Y.S. Lai and C.R. Kao: Electronic Components and Technology Conference, (2011) pp. 1481-1488.

5) J.S. Cho, H.S. Jeong, J.T. Moon, S.J. Yoo, J.S. Seo, S.M. Lee, S.W. Ha, E.K. Her, S.H. Kang and K.H. Oh: Electronic Components and Technology Conference, (2009) pp. 1569-1573.

6) H. Lio, O. Chen, Z. Zhao, Q. Wang, J. Zeng, J. Chae and J. Lee: Electronic Components and Technology Conference, (2010) pp. 234239.

7) C.-H. Chuang, C.-H. Tsai, Y.-C. Lin and H.-J. Lin: Metals 6 (2016) 182 .

8) J.K. Liao, L.Y. Hung, L.W. Wu and Y.P. Wang: Electronic Components and Technology Conference, (2012) pp. 1163-1168.

9) N. Murali, N. Srikanth and C.J. Vath, III: Mater. Charact. 50 (2003) 39-50.

10) S.J. Hong, J.S. Cho, J.T. Moon and J. Lee: International Symposium on Electronic Materials and Packaging, (2001) pp. 52-55.

11) J.L. Chen and Y.C. Lin: IEEE Trans. Electron. Packag. Manuf. 23 (2000) 116-122.

12) J. Onuki, M. Koizumi and H. Suzuki: J. Appl. Phys. 68 (1990) 56105614.

13) T. Jonathan, H.T. Boon and M.H. Hong: Electronics Packaging Technology Conference, (2004) pp. 711-717.

14) H.-W. Hsueh, F.-Y. Hung, T.-S. Lui, L.-H. Chen and K.-J. Chen: Adv. Mater. Sci. Eng. (2014) 1-6. 